PROCEEDINGS OF THE

AMERICAN MATHEMATICAL SOCIETY

Volume 127, Number 8, Pages 2385-2393

S 0002-9939(99)04895-9

Article electronically published on April 9, 1999

\title{
BCL INDEX AND FREDHOLM TUPLES
}

\author{
RONGWEI YANG
}

(Communicated by Theodore W. Gamelin)

\begin{abstract}
In this paper we will prove that the BCL index for $C^{*}$-algebras generated by two essentially doubly commuting isometries is equal to the index of the Fredholm tuples formed by these two isometries. We will then compute this index for certain sub-Hardy modules over the bidisk. Some interesting corollaries are also listed.
\end{abstract}

\section{INTRODUCTION}

In [BCL], Berger, Coburn and Lebow made a study of the $C^{*}$-algebra generated by commuting isometries. In section 5 of their paper, the authors were able to define an index for the $C^{*}$-algebra generated by two essentially doubly commuting isometries. They conjectured that this index is a unitary invariant for the algebra and various cases were discussed in detail.

In the first two sections of this paper, we will compute the index and show that it is equal to the index of a specific Fredholm tuple. In section 3 we will compute the index for certain sub-Hardy modules over the bidisk.

We begin by introducing some notations and a lemma from [BCL].

Let $H$ be a separable Hilbert space and $V_{1}, V_{2}$ be two commuting isometries acting on $H . H_{0}$ is the subspace defined by

$$
H_{0}:=\left\{x \in H: x=\left(V_{1} V_{2}\right)^{n} y_{n} \text { for all } n>0\right\}=\bigcap_{n=1}^{\infty}\left(V_{1} V_{2}\right)^{n} H .
$$

Then $H_{0}$ reduces $V_{1} V_{2}$ and $H^{\perp}=0$ or $H^{\perp}$ is unitarily eqvivalent to $H^{2}(T) \otimes$ $H^{\prime}$ where $H^{2}(T)$ is the Hardy space and $H^{\prime}$ some separable Hilbert space, and $\left.V_{1} V_{2}\right|_{H^{\perp}} \cong T_{z} \otimes I$ under this unitary equivalence. We have the following lemma from $[\mathrm{BCL}]$.

Lemma 0.1. If $V_{1}, V_{2}$ satisfy the hypotheses: $H_{0}=0, H^{\prime}$ infinite dimensional and the commutator $\left[V_{2}^{*}, V_{1}\right]$ compact, then for

$$
C\left(V_{1}, V_{2}\right):=\left[V_{2}^{*}, V_{2}\right]\left(V_{1}-I\right)\left[V_{2}^{*}, V_{2}\right],
$$

$I+C\left(V_{1}, V_{2}\right)$ is either Fredholm or semi-Fredholm of index $-\infty$.

Received by the editors November 3, 1997.

1991 Mathematics Subject Classification. Primary 47C10; Secondary 47B35.

(C)1999 American Mathematical Society 


\section{BCL INDEX}

In this section, we will compute ind $\left(I+C\left(V_{1}, V_{2}\right)\right)$ under the hypotheses of Lemma 0.1 .

We first observe that $V_{1}$ essentially commutes with $\left[V_{2}^{*}, V_{2}\right]$ and that

$$
\left[V_{2}^{*}, V_{2}\right]^{2}=\left[V_{2}^{*}, V_{2}\right]
$$

Therefore,

$$
\begin{aligned}
\operatorname{ind}\left(I+C\left(V_{1}, V_{2}\right)\right) & =\operatorname{ind}\left(I+\left(V_{1}-I\right)\left[V_{2}^{*}, V_{2}\right]^{2}\right) \\
& =\operatorname{ind}\left(I+\left(V_{1}-I\right)\left[V_{2}^{*}, V_{2}\right]\right) .
\end{aligned}
$$

We let $D$ denote the operator $I+\left(V_{1}-I\right)\left[V_{2}^{*}, V_{2}\right]$.

Lemma 1.1. $D(H)=V_{1} H+V_{2} H$.

Proof. For any $x \in H$,

$$
\begin{aligned}
D x & =x+\left(V_{1}-I\right)\left(I-V_{2} V_{2}^{*}\right) x \\
& =x+\left(V_{1}-I\right) x-\left(V_{1}-I\right) V_{2} V_{2}^{*} x=V_{1} x-V_{2}\left(V_{1}-I\right) V_{2}^{*} x .
\end{aligned}
$$

This implies that

$$
D(H) \subset V_{1} H+V_{2} H .
$$

On the other hand,

$$
\begin{aligned}
D V_{2} x & =V_{2} x+\left(V_{1}-I\right) V_{2} x-\left(V_{1}-I\right) V_{2} V_{2}^{*} V_{2} x \\
& =V_{2} x+\left(V_{1}-I\right) V_{2} x-\left(V_{1}-I\right) V_{2} x=V_{2} x .
\end{aligned}
$$

This shows that $V_{2}(H) \subset D(H)$. Furthermore,

$$
D x+V_{2}\left(V_{1}-I\right) V_{2}^{*} x=x+\left(V_{1}-I\right) x=V_{1} x .
$$

So $V_{1}(H) \subset D(H)$ and hence, $V_{1} H+V_{2} H \subset D(H)$.

By (1-1), the kernel of $D$ are the solutions of the equation

$$
V_{1} x=\left(V_{1}-I\right) V_{2} V_{2}^{*} x .
$$

Suppose $x$ is a solution of this equation. We write

$$
x=x_{1}+x_{2}
$$

with $x_{1} \in H \ominus V_{2} H$ and $x_{2} \in V_{2} H$. Then,

$$
\begin{aligned}
V_{1} x=V_{1}\left(x_{1}+x_{2}\right) & =\left(V_{1}-I\right) V_{2} V_{2}^{*} x_{1}+\left(V_{1}-I\right) V_{2} V_{2}^{*} x_{2} \\
& =\left(V_{1}-I\right) V_{2} V_{2}^{*} x_{1}+\left(V_{1}-I\right) x_{2} .
\end{aligned}
$$

This implies that

$$
V_{1} x_{1}=\left(V_{1}-I\right) V_{2} V_{2}^{*} x_{1}-x_{2},
$$

and hence,

$$
x_{2}=\left(V_{1}-I\right) V_{2} V_{2}^{*} x_{1}-V_{1} x_{1},
$$

in particular, $V_{1} x_{1} \in V_{2}(H)$ and $x_{2}$ is uniquely determined by $x_{1}$. Conversely, let $x_{1}$ be in $H \ominus V_{2}(H)$ such that $V_{1} x_{1} \in V_{2}(H)$. We let $x_{2}$ be determined by equation 
(1-3) and $x=x_{1}+x_{2}$. One sees that $x_{2} \in V_{2}(H)$ and that

$$
\begin{aligned}
& \left(V_{1}-I\right) V_{2} V_{2}^{*} x \\
& =\left(V_{1}-I\right) V_{2} V_{2}^{*}\left(x_{1}+x_{2}\right) \\
& =\left(V_{1}-I\right) V_{2} V_{2}^{*} x_{1}+\left(V_{1}-I\right) x_{2} \\
& =\left(V_{1}-I\right) V_{2} V_{2}^{*} x_{1}+\left(V_{1}-I\right)\left[\left(V_{1}-I\right) V_{2} V_{2}^{*} x_{1}-V_{1} x_{1}\right] \\
& =\left(V_{1}-I\right) V_{2} V_{2}^{*} x_{1}+V_{1}\left(V_{1}-I\right) V_{2} V_{2}^{*} x_{1}-\left(V_{1}-I\right) V_{2} V_{2}^{*} x_{1}-\left(V_{1}-I\right) V_{1} x_{1} \\
& =V_{1}\left[x_{1}+\left(V_{1}-I\right) V_{2} V_{2}^{*} x_{1}-V_{1} x_{1}\right] \\
& =V_{1}\left(x_{1}+x_{2}\right)=V_{1} x .
\end{aligned}
$$

Combining all these observations we have the following:

Lemma 1.2. The projection from $H$ onto $H \ominus V_{2}(H)$ induces an invertible map from $\operatorname{Ker}(D)$ to the space

$$
H_{1}:=\left\{x \in H \ominus V_{2}(H): V_{1} x \in V_{2}(H)\right\} .
$$

Combining Lemmas 0.1, 1.1 and 1.2 we have

Theorem 1.3. Suppose $V_{1}, V_{2}$ are isometries satisfying the hypotheses in Lemma 0.1 , then $\operatorname{dim} H_{1}<\infty$ and

$$
\operatorname{ind}\left(I+C\left(V_{1}, V_{2}\right)=\operatorname{ind}(D)=\operatorname{dim} H_{1}-\operatorname{dim}\left(H \ominus\left(V_{1}(H)+V_{2}(H)\right)\right) .\right.
$$

Though it seems that this index is dependent on the order of the pair $\left(V_{1}, V_{2}\right)$, it is actually not. In fact, if we let

$$
H_{2}:=\left\{x \in H \ominus V_{1}(H): V_{2} x \in V_{1}(H)\right\},
$$

then one can check easily that $V_{2}^{*} V_{1}$ is unitary from $H_{1}$ to $H_{2}$ and thus, $\operatorname{dim} H_{1}=$ $\operatorname{dim} \mathrm{H}_{2}$.

\section{FredHolm tUPLES}

Let $a, b$ be two commuting isometries acting on $H$ and $d_{1}$ be the map from $H$ to $H \oplus H$, such that

$$
d_{1} x=(-b x, a x), \quad x \in H
$$

and $d_{2}$ the map from $H \oplus H$ to $H$ such that

$$
d_{2}(x, y)=a x+b y, \quad x, y \in H .
$$

Then one checks that

$$
d_{2} d_{1}=0 .
$$

We consider the following short sequence:

$$
0 \rightarrow H \stackrel{d_{1}}{\rightarrow} H \oplus H \stackrel{d_{2}}{\rightarrow} H \rightarrow 0 .
$$

The tuple $(a, b)$ is called Fredholm if

$$
\operatorname{dim}\left(\operatorname{Ker}\left(d_{1}\right)\right)+\operatorname{dim}\left(\operatorname{Ker}\left(d_{2}\right) \ominus d_{1}(H)\right)+\operatorname{dim}\left(H \ominus d_{2}(H \oplus H)\right)<+\infty
$$

and is called semi-Fredholm if no more than one of the homology spaces is infinite dimensional. The index of the tuple $(a, b)$ is defined by

$$
\operatorname{ind}(a, b):=-\operatorname{dim}\left(\operatorname{Ker}\left(d_{1}\right)\right)+\operatorname{dim}\left(\operatorname{Ker}\left(d_{2}\right) \ominus d_{1}(H)\right)-\operatorname{dim}\left(H \ominus d_{2}(H \oplus H)\right) .
$$

Now we have a look at the case when $a, b$ are both isometries. It is not hard to see that $\operatorname{Ker}\left(d_{1}\right)=0$ and $d_{2}(H \oplus H)=a(H)+b(H)$. 


\section{Lemma 2.1.}

$$
\operatorname{Ker}\left(d_{2}\right) \ominus d_{1}(H)=\left\{\left(x,-b^{*} a x\right): x \in H \ominus b(H) \text { and } a x \in b(H)\right\} .
$$

Proof. First of all, if $x$ is as in the right-hand side of (2-2), then

$$
d_{2}\left(x,-b^{*} a x\right)=a x-b b^{*} a x=a x-a x=0,
$$

and for any $y \in H$,

$$
\begin{aligned}
\left\langle\left(x,-b^{*} a x\right), d_{1} y\right\rangle & =\langle x,-b y\rangle+\left\langle-b^{*} a x, a y\right\rangle \\
& =\langle x,-b y\rangle-\langle a x, b a y\rangle \\
& =\langle x,-b y\rangle-\langle a x, a b y\rangle=-2\langle x, b y\rangle=0 .
\end{aligned}
$$

This shows that the right-hand side of (2-2) is contained in the left-hand side. On the other hand, if $(x, z)$ is an element of the left-hand side, then

$$
0=d_{2}(x, z)=a x+b z,
$$

and hence, $z=-b^{*} a x$. That $x$ is in $H \ominus b(H)$ is also implied by (2-3).

Lemma 2.1 and Theorem 1.3 together imply the following

Theorem 2.2. If $V_{1}, V_{2}$ are two commuting isometries that satisfy the hypotheses in Lemma 0.1, then the tuple $\left(V_{1}, V_{2}\right)$ is semi-Fredholm and

$$
\operatorname{ind}\left(I+C\left(V_{1}, V_{2}\right)\right)=\operatorname{ind}\left(V_{1}, V_{2}\right) \text {. }
$$

\section{Fredholm tuples ON SUB-HARdy MOdUles}

Let $H^{2}\left(T^{2}\right)$ denote the Hardy space over the torus. It is well known that it is an $A\left(D^{2}\right)$ module, where $A\left(D^{2}\right)$ stands for the bidisc algebra. A submodule of $H^{2}\left(T^{2}\right)$ is a closed subspace that is invariant under the module action by $A\left(D^{2}\right)$. For any $h \in H^{2}\left(T^{2}\right)$, we let $[h]$ denote the submodule generated by $h$ and $H^{2}\left(T^{2}\right) \ominus[h]$ denote the quotient module. For any submodule $H, R_{1}$ and $R_{2}$ will denote the restrictions of the multiplications by the coordinate functions $z_{1}$ and $z_{2}$ to $H$. One sees that $R_{1}, R_{2}$ are commuting isometries. When $H$ is generated by a finite number of polynomials, a result in [Ya] shows that the commutator $\left[R_{1}^{*}, R_{2}\right]$ is Hilbert-Schmidt. Therefore, $R_{1}, R_{2}$ satisfy the hypotheses of Lemma 0.1 . In this section we will make a study of the tuple $\left(R_{1}, R_{2}\right)$.

Lemma 3.1. If $H \subset H^{2}\left(T^{2}\right)$ is a submodule, then

$$
\operatorname{rank}(H) \geq \operatorname{dim}\left(H \ominus\left(z_{1} H+z_{2} H\right)\right) .
$$

Proof. Suppose $\operatorname{rank}(H)=k<\infty$. Then we can choose $f_{1}, f_{2}, \ldots, f_{k}$ such that $H=\left[f_{1}, f_{2}, \ldots, f_{k}\right]$. If $\operatorname{rank}(H)<\operatorname{dim}\left(H \ominus\left(z_{1} H+z_{2} H\right)\right)$, we can find a nonzero $f \in H \ominus\left(z_{1} H+z_{2} H\right)$ such that it is orthogonal to $p f_{j}$ for each $j$, where $p$ is the projection from $H$ to $H \ominus\left(z_{1} H+z_{2} H\right)$. This will imply that $f$ is orthogonal to each $f_{j}$ and hence it is orthogonal to $f_{j}\left(z_{1}, z_{2}\right) p\left(z_{1}, z_{2}\right)=f_{j}\left(z_{1}, z_{2}\right) p(0,0)+$ $f_{j}\left(z_{1}, z_{2}\right)\left(p\left(z_{1}, z_{2}\right)-p(0,0)\right)$ for every polynomial $p$. In other words, $f$ is orthogonal to $H$ which is impossible unless $f=0$.

While it is not hard to see that the equality in Lemma 3.1 holds when $H$ is singly generated, we are unable to find a tool to handle the problem in general. But we believe the equality in Lemma 3.1 is true at least for some other nicely generated submodules if not for all. 
Conjecture. The equality in Lemma 3.1 holds for every submodule generated by polynomials.

We will study this conjecture at a later time.

Corollary 3.2. If $H$ is generated by finitely many polynomials, then $\left(R_{1}, R_{2}\right)$ is Fredholm on $H$ and ind $\left(R_{1}, R_{2}\right)=-1$.

Proof. First of all $\left[R_{1}^{*}, R_{2}\right]$ is compact by Corollary 6.2 in [Ya]. One checks further that $\left(R_{1}, R_{2}\right)$ satisfies the conditions in Lemma 0.1. The semi-Fredholmness of $\left(R_{1}, R_{2}\right)$ is then implied by Theorem 2.2 .

We now assume that $H$ is generated by one polynomial, say $h$. Then by Lemma 3.1 it is not hard to see that

$$
\operatorname{dim}\left(H \ominus\left(z_{1} H+z_{2} H\right)\right)=1 .
$$

So by Theorems 1.3 and 2.2, it suffices to show that $H_{1}=0$. In fact, if $f \in H_{1}$, we can write $f=h g$ for some holomorphic function $g$. Then,

$$
z_{1} h g=z_{2} h \phi
$$

for some $h \phi \in H$. Therefore,

$$
z_{1} g=z_{2} \phi
$$

hence, $g$ has the factor $z_{2}$ which implies that $f$ is also in $z_{2} H$ and this is possible only if $f=0$.

If $H$ is generated by $k$ polynomials, say $h_{1}, h_{2}, \ldots, h_{k}$, then $[G] \ominus H$ is finite dimensional following from Lemma 6.1 in [Ya], where $G=G C D\left(h_{1}, h_{2}, \ldots, h_{k}\right)$ and $[G]$ denotes the sub-module generated by $G$. This means that the tuple $\left(R_{1}, R_{2}\right)$ on $H$ is a compact perturbation of the tuple $\left(R_{1}, R_{2}\right)$ on $[G]$ and therefore, these two tuples have the same index.

It is possible to give a more direct proof of this corollary by a study of the $C^{*}$ algebra generated by the two commuting isometries $R_{1}$ and $R_{2}$. Interested readers may find useful tools in [BCL] and [Do] to this end.

Let $H$ be any submodule of $H^{2}\left(T^{2}\right)$ and let $q: H^{2}\left(T^{2}\right) \longrightarrow H^{2}\left(T^{2}\right) \ominus H$ be the projection onto the quotient space. $H^{2}\left(T^{2}\right) \ominus H$ is the quotient $A\left(D^{2}\right)$ module with the module action of $A\left(D^{2}\right)$ on $H^{2}\left(T^{2}\right)$ compressed by $q$; more specifically,

$$
f \cdot x:=q f x,
$$

where $f \in A\left(D^{2}\right)$ and $x \in H^{2}\left(T^{2}\right) \ominus H$.

We let $S_{1}:=q z_{1}, S_{2}:=q z_{2}$. It is then easy to see how the tuple $\left(S_{1}, S_{2}\right)$ on $H^{2}\left(T^{2}\right) \ominus H$ is related to the tuple $\left(R_{1}, R_{2}\right)$ on $H$. The following part of this paper is devoted to this question.

Let $H$ be any submodule of $H^{2}\left(T^{2}\right)$ and $H_{1}, H_{2}$ be as in section 1.

Lemma 3.3. $\overline{z_{2}} H_{1}=\overline{z_{1}} H_{2}$.

Proof. First of all, from the definition of $H_{1}, H_{2}$, it is not hard to see that

$$
\overline{z_{2}} H_{1} \subset H^{2}\left(T^{2}\right) ; \quad \overline{z_{1}} H_{2} \subset H^{2}\left(T^{2}\right) .
$$

By the remarks that immediately follow Theorem 1.3 we also have that

$$
z_{1} \overline{z_{2}} H_{1}=\overline{z_{2}} z_{1} H_{1}=H_{2} .
$$

Therefore,

$$
\overline{z_{1}} H_{2}=\overline{z_{2}} H_{1} \text {. }
$$


If we denote $\overline{z_{2}} H_{1}$ by $E$, then $\operatorname{dim} E=\operatorname{dim} H_{1}=\operatorname{dim} H_{2}$. The following proposition gives a more concrete discription of the space $E$.

Proposition 3.4. $E=\operatorname{Ker}\left(S_{1}\right) \cap \operatorname{Ker}\left(S_{2}\right)$.

Proof. We let $q: H^{2}\left(T^{2}\right) \longrightarrow H^{2}\left(T^{2}\right) \ominus H$ be the projection. Then,

$$
S_{1}=q z_{1} ; \quad S_{2}=q z_{2} .
$$

Therefore,

$$
\begin{aligned}
& S_{1}(E)=q z_{1}\left(\overline{z_{1}} H_{2}\right)=q\left(H_{2}\right)=0 ; \\
& S_{2}(E)=q z_{2}\left(\overline{z_{2}} H_{1}\right)=q\left(H_{1}\right)=0 .
\end{aligned}
$$

Conversely, if $f \in \operatorname{Ker}\left(S_{1}\right) \cap \operatorname{Ker}\left(S_{2}\right)$, then

$$
S_{1} f=S_{2} f=0 .
$$

This implies that $z_{1} f, z_{2} f$ are both in $H$. Since $f$ is orthogonal to $H$, we actually have that

$$
z_{1} f \in H \ominus z_{1} H ; \quad z_{2} f \in H \ominus z_{2} H .
$$

Furthermore,

$$
z_{1} z_{2} f=z_{2} z_{1} f \in z_{1} H \cap z_{2} H
$$

hence, $z_{1} f \in H_{2}$ and $z_{2} f \in H_{1}$, i.e.,

$$
f \in E=\overline{z_{1}} H_{2}=\overline{z_{2}} H_{1} .
$$

Corollary 3.5. If $H$ is a submodule such that $\left[R_{1}^{*}, R_{2}\right]$ is compact on $H$, then $\operatorname{dim}\left(\operatorname{Ker}\left(S_{1}\right) \cap \operatorname{Ker}\left(S_{2}\right)\right)<\infty$ and

$$
\operatorname{ind}\left(R_{1}, R_{2}\right)=\operatorname{dim}\left(\operatorname{Ker}\left(S_{1}\right) \cap \operatorname{Ker}\left(S_{2}\right)\right)-\operatorname{dim}\left(\operatorname{Ker}\left(R_{1}^{*}\right) \cap \operatorname{Ker}\left(R_{2}^{*}\right)\right) .
$$

Proof. This follows directly from Theorem 1.3, the above proposition and the fact that $H \ominus\left(z_{1} H+z_{2} H\right)=\operatorname{Ker}\left(R_{1}^{*}\right) \cap \operatorname{Ker}\left(R_{2}^{*}\right)$.

It would be very interesting to give a direct proof of this fact.

Lemma (Curto $[\mathrm{Cu}])$. If $(a, b)$ is a doubly commuting tuple on $H$, and $v$ is a Fredholm operator on $H$ that commutes with both $a, b$, then $(v a, b)$ is Fredholm if $(a, b)$ is.

Suppose $\lambda=\left(\lambda_{1}, \lambda_{2}\right) \in D^{2}$. We let

$$
\phi_{1}=\frac{z_{1}-\lambda_{1}}{1-\overline{\lambda_{1}} z_{1}}, \phi_{2}=\frac{z_{2}-\lambda_{2}}{1-\overline{\lambda_{2}} z_{2}} .
$$

Then $\phi_{1}, \phi_{2}$ are inner functions in different variables, so $R_{\phi_{1}}$ and $R_{\phi_{2}}$ are two commuting isometries and $\left[R_{\phi_{1}}^{*}, R_{\phi_{2}}\right.$ ] is compact by [Ya], Lemma 1.3 and [Ya], Corollary 6.2 when $H$ is generated by a finite number of polynomials. One further checks that $R_{\phi_{1}}, R_{\phi_{2}}$ satisfy the hypotheses of Lemma 0.1 . Hence, $\left(R_{\phi_{1}}, R_{\phi_{2}}\right)$ is Fredholm by Theorems 1.3 and 2.2. Now we use this fact and Curto's lemma to prove the following

Theorem 3.6. If $H$ is a submodule generated by finitely many polynomials, then $R_{\lambda}=\left(R_{1}-\lambda_{1}, R_{2}-\lambda_{2}\right)$ is Fredholm for any $\lambda=\left(\lambda_{1}, \lambda_{2}\right) \in D^{2}$ and $\operatorname{ind}\left(R_{\lambda}\right)=-1$. 
Proof. We observe that

$$
R_{\phi_{1}}=\left(1-\overline{\lambda_{1}} R_{1}\right)^{-1}\left(R_{1}-\lambda_{1}\right) ; R_{\phi_{2}}=\left(1-\overline{\lambda_{2}} R_{2}\right)^{-1}\left(R_{2}-\lambda_{2}\right) .
$$

Curto's lemma and the remarks following it imply that $R_{\lambda}=\left(R_{1}-\lambda_{1}, R_{2}-\lambda_{2}\right)$ is Fredholm. Furthermore, $R_{\lambda}$ is connected to $\left(R_{1}, R_{2}\right)$ by the path $R_{t \lambda}, 0 \leq t \leq 1$. Hence,

$$
\operatorname{ind}\left(R_{\lambda}\right)=\operatorname{ind}\left(R_{1}, R_{2}\right)=-1
$$

Corollary 3.7. If $H$ is a submodule generated by polynomials, then

$$
\operatorname{dim}\left(\operatorname{Ker}\left(S_{1}\right) \cap \operatorname{Ker}\left(S_{2}\right)\right)=\operatorname{dim}\left(H \ominus\left(z_{1} H+z_{2} H\right)\right)-1 .
$$

Proof. This follows easily from Theorem 1.3, Corollary 3.5 and the above theorem.

Lemma 3.8. If $H$ is a submodule and $K=H^{2}\left(T^{2}\right) \ominus H$, then

$$
\operatorname{dim}\left(\operatorname{Ker}\left(S_{1}\right) \cap \operatorname{Ker}\left(S_{2}^{*}\right)\right)<\infty ; \operatorname{dim}\left(\operatorname{Ker}\left(S_{2}\right) \cap \operatorname{Ker}\left(S_{1}^{*}\right)\right)<\infty .
$$

Proof. We prove the first inequality.

Suppose $f$ is any function in $\operatorname{Ker}\left(S_{1}\right) \cap \operatorname{Ker}\left(S_{2}^{*}\right)$. First of all, $\overline{z_{2}} f$ is orthogonal to $H$ since $f$ is in $K$. On the other hand,

$$
q \overline{z_{2}} f=S_{2}^{*} f=0,
$$

so $\overline{z_{2}} f$ is also orthogonal to $K$. This implies that $\overline{z_{2}} f$ is orthogonal to $H^{2}\left(T^{2}\right)$ which means that $f$ belongs in $H^{2}\left(T^{2}\right) \ominus z_{2} H^{2}\left(T^{2}\right)$. In other words, $f$ is independent of $z_{2}$ and hence $z_{1} f$ is also in $H^{2}\left(T^{2}\right) \ominus z_{2} H^{2}\left(T^{2}\right)$.

Furthermore, since $S_{1} f=0$, one checks easily that $z_{1} f \in H \ominus z_{1} H$. Now assume $\left\{f_{n}: n=1,2, \ldots, l\right\}(l$ can be $\infty)$ is an orthogonal basis for $\operatorname{Ker}\left(S_{1}\right) \cap \operatorname{Ker}\left(S_{2}^{*}\right)$. The arguments above say that for each $n$,

$$
z_{1} f_{n} \subset\left(H \ominus z_{1} H\right) \cap\left(H^{2}\left(T^{2}\right) \ominus z_{2} H^{2}\left(T^{2}\right)\right) .
$$

Assume $N_{\lambda}$ is a mapping from $H^{2}\left(T^{2}\right)$ to $H^{2}(T)$ such that for any $f(z, w)=$ $\sum_{k=0}^{\infty} f_{k}(z) w^{k} \in H^{2}\left(T^{2}\right)$,

$$
N_{\lambda} f(z)=f(z, \lambda) .
$$

By Corollary 1.2 in [Ya], $N_{\lambda}$ is Hilbert-Schmidt on $H \ominus z_{1} H$. And by the previous remarks, $z_{1} f_{n}$ is independent of $z_{2}$ for each $n$. Therefore,

$$
N_{\lambda}\left(z_{1} f_{n}\right)=z_{1} f_{n},
$$

and

$$
\infty>\sum_{n=1}^{l}\left\|N_{\lambda}\left(z_{1} f_{n}\right)\right\|^{2}=\sum_{n=1}^{l}\left\|\left(z_{1} f_{n}\right)\right\|^{2}=\sum_{n=1}^{l} 1=l .
$$

Proposition 3.9. If $\left[S_{1}^{*}, S_{2}\right]$ is compact on $K=H^{2}\left(T^{2}\right) \ominus H$, then the tuple $\left(S_{1}, S_{2}\right)$ is Fredholm on $K$ if $\left(R_{1}, R_{2}\right)$ is Fredholm on $H$. 
Proof. By Corollary 3.8 in $[\mathrm{Cu}],\left(S_{1}, S_{2}\right)$ is Fredholm if and only if

$$
\tilde{S}:=\left(\begin{array}{cc}
S_{1} & S_{2} \\
-S_{2}^{*} & S_{1}^{*}
\end{array}\right)
$$

is Fredholm on $K \oplus K$. Since

$$
\tilde{S} \tilde{S}^{*}:=\left(\begin{array}{cc}
S_{1} S_{1}^{*}+S_{2} S_{2}^{*} & 0 \\
0 & S_{1}^{*} S_{1}+S_{2}^{*} S_{2}
\end{array}\right),
$$

we have that

$$
\operatorname{Ker}\left(\tilde{S}^{*}\right)=\left(k e r\left(S_{1}^{*}\right) \cap \operatorname{Ker}\left(S_{2}^{*}\right)\right) \oplus\left(k e r\left(S_{1}\right) \cap \operatorname{Ker}\left(S_{2}\right)\right) .
$$

If $\left(R_{1}, R_{2}\right)$ is Fredholm on $H$, then

$$
\operatorname{dim}\left(\operatorname{Ker}\left(d_{2}\right) \ominus d_{1}(H)\right)<\infty
$$

by formula (2-1). But by Lemma 2.1 ,

$$
\operatorname{Ker}\left(d_{2}\right) \ominus d_{1}(H)=H_{1} ;
$$

Proposition 3.4 then implies that

$$
\operatorname{dim}\left(\operatorname{ker}\left(S_{1}\right) \cap \operatorname{Ker}\left(S_{2}\right)\right)<\infty .
$$

Furthermore, from the proof of Lemma 3.8,

$$
\operatorname{Ker}\left(S_{1}^{*}\right) \subset H^{2}\left(T^{2}\right) \ominus z_{1} H^{2}\left(T^{2}\right), \quad \operatorname{Ker}\left(S_{2}^{*}\right) \subset H^{2}\left(T^{2}\right) \ominus z_{2} H^{2}\left(T^{2}\right) .
$$

Hence,

$$
\operatorname{dim}\left(\operatorname{ker}\left(S_{1}^{*}\right) \cap \operatorname{Ker}\left(S_{2}^{*}\right)\right) \leq \operatorname{dim}\left(H^{2}\left(T^{2}\right) \ominus\left(z_{1} H^{2}\left(T^{2}\right)+z_{2} H^{2}\left(T^{2}\right)\right)=1 .\right.
$$

So $\operatorname{dim}\left(\operatorname{Ker}\left(\tilde{S}^{*}\right)\right)$ is finite.

We now deal with $\operatorname{Ker}(\tilde{S})$. First, one computes that

$$
\tilde{S}^{*} \tilde{S}:=\left(\begin{array}{cc}
S_{1}^{*} S_{1}+S_{2} S_{2}^{*} & S_{1}^{*} S_{2}-S_{2} S_{1}^{*} \\
S_{2}^{*} S_{1}-S_{1} S_{2}^{*} & S_{1} S_{1}^{*}+S_{2}^{*} S_{2}
\end{array}\right) .
$$

But $\left[S_{1}^{*}, S_{2}\right]$, and hence $\left[S_{2}^{*}, S_{1}\right]$, is compact from the assumption, $\operatorname{Ker}\left(\tilde{S}^{*} \tilde{S}\right)$ is finite dimensional if and only if

$$
A:=\left(\begin{array}{cc}
S_{1}^{*} S_{1}+S_{2} S_{2}^{*} & 0 \\
0 & S_{1} S_{1}^{*}+S_{2}^{*} S_{2}
\end{array}\right)
$$

has finite dimensional kernel, but this is already implied by Lemma 3.8.

In conclusion, $\tilde{S}$ is Fredholm on $K \oplus K$ and so is $\left(S_{1}, S_{2}\right)$.

Corollary 3.10. If $H$ is generated by polynomials, then the tuple $\left(S_{1}, S_{2}\right)$ is Fredholm on $K=H^{2}\left(T^{2}\right) \ominus H$.

Proof. This follows directly from Corollary 6.2 in [Ya] and the above proposition.

It would be interesting to compute the index of the tuple $\left(S_{1}, S_{2}\right)$ and see how it is related to the $C^{*}$-algebra generated by $S_{1}$ and $S_{2}$.

\section{ACKNOWLEDGEMENT}

The author thanks Prof. Ronald G. Douglas for many helpful discussions. 


\section{REFERENCES}

[BCL] C. Berger, L. Coburn, A. Lebow, Representation and index theory for $C^{*}$-algebras generated by commuting isometries, J. Functional Analysis 27 (1978), No. 1, 51-99. MR 57:7251

[Cu] R. Curto, Fredholm and invertible tuples of bounded linear operators, thesis, Math. Dept. of SUNY-Stonybrook 1978.

[Do] R. Douglas, Local Toeplitz operators, Proc. London Math. Soc. 36 (1978), 243-272. MR 58:2421

[Ya] R. Yang, The Berger-Shaw theorem in Hardy modules over the bidisk, submitted to J. of Operator Theory.

Department of Mathematics, Texas A\&M University, College Station, Texas 77843

E-mail address: ryang@math.tamu.edu

Department of Mathematics, SUny at Stony Brook, Stony Brook, New York 11794 